\title{
FOURTH ORDER GEOMETRIC EVOLUTION EQUATIONS
}

\author{
GLEN WHEELER
}

(Received 20 September 2010) 2010 Mathematics subject classification: primary 35K59; secondary 35G20, 53B20, 53C21, 53C44,
58J35.

Keywords and phrases: evolution equations, hypersurface flows.

In this thesis the chief objects of study are hypersurface flows of fourth order, with the speed of the flow varying from the Laplacian of the mean curvature, to the more general constrained flows which include a function of time in the speed, and satisfy various conditions. Our aim is to instigate a study of the regularity of these flows, answering questions of local and global existence, and some preliminary singularity analysis. Among our results are positive lower bounds for smooth and regular existence, classification of stationary solutions, interior estimates, and blowup asymptotics. Applying these results to a certain class of constrained surface diffusion flows, we obtain long time existence and exponential convergence to spheres for initial surfaces with small $L^{2}$ norm of tracefree curvature. We present one application of this theorem, using it to deduce the isoperimetric inequality with optimal constant for 2-surfaces satisfying the above smallness condition. The long time existence theorem can be thought of as a stability of spheres result, as the smallness condition is an averaged distance from a standard round sphere to the initial manifold in $L^{2}$. This strengthens a related earlier result specialized to the surface diffusion and Willmore flows [17], where the distance is small in $C^{2, \alpha}$, obtained through a completely different method. Our techniques have more in common with [9-11], from which we have drawn much inspiration. The results throughout this thesis are new contributions for both surface diffusion flow, which has been considered by many authors $[1-8,12,16,17]$, and the constrained flows, which have only recently been considered [13-15, 18, 19].

Thesis submitted to the University of Wollongong, February 2010. Degree approved, July 2010. Supervisors: Dr James McCoy and Professor Graham Williams.

(C) 2010 Australian Mathematical Publishing Association Inc. 0004-9727/2010 \$16.00 


\section{References}

[1] P. Baras, J. Duchon and R. Robert, 'Evolution d'une interface par diffusion de surface', Comm. Partial Differential Equations 9(4) (1984), 313-335.

[2] J. W. Cahn, C. M. Elliott and A. Novick-Cohen, 'The Cahn-Hilliard equation with a concentration dependent mobility: motion by minus the Laplacian of the mean curvature', European J. Appl. Math. 7(3) (1996), 287-301.

[3] J. W. Cahn and J. E. Taylor, 'Surface motion by surface diffusion', Acta Metall. Mater. 42(4) (1994), 1045-1063.

[4] F. Davì and M. E. Gurtin, 'On the motion of a phase interface by surface diffusion', Z. Angew. Math. Phys. 41(6) (1990), 782-811.

[5] C. M. Elliott and H. Garcke, 'Existence results for diffusive surface motion laws', Adv. Math. Sci. Appl. 7(1) (1997), 465-488.

[6] J. Escher, U. F. Mayer and G. Simonett, 'The surface diffusion flow for immersed hypersurfaces', SIAM J. Math. Anal. 29(6) (1998), 1419-1433.

[7] P. C. Fife, 'Models for phase separation and their mathematics', Electron. J. Differential Equations 2000(48) (2000), 1-26.

[8] K. Ito, 'The surface diffusion flow equation does not preserve the convexity', RIMS Kôkyâroku Bessatsu 1105 (1999), 10-21.

[9] E. Kuwert and R. Schatzle, 'The Willmore flow with small initial energy', J. Differential Geom. 57(3) (2001), 409-441.

[10] E. Kuwert and R. Schatzle, 'Gradient flow for the Willmore functional', Comm. Anal. Geom. 10(2) (2002), 307-339.

[11] E. Kuwert and R. Schatzle, 'Removability of point singularities of Willmore surfaces', Ann. of Math. (2) 160 (2004), 315-357.

[12] U. F. Mayer, 'Numerical solutions for the surface diffusion flow in three space dimensions', J. Comput. Appl. Math. 20(3) (2001), 361-379.

[13] J. McCoy, 'The surface area preserving mean curvature flow', Asian J. Math. 7(1) (2003), 7-30.

[14] J. McCoy, 'The mixed volume preserving mean curvature flow', Math. Z. 246(1-2) (2004), $155-166$.

[15] J. McCoy, 'Mixed volume preserving curvature flows', Calc. Var. Partial Differential Equations 24(2) (2005), 131-154.

[16] W. W. Mullins, 'Theory of thermal grooving', J. Appl. Phys. 28 (1957), 333-339.

[17] G. Simonett, 'The Willmore flow for near spheres', Differential Integral Equations 14(8) (2001), $1005-1014$.

[18] G. Wheeler, 'Lifespan theorem for simple constrained surface diffusion flows'. J. Math. Anal. Appl. (2010), to appear.

[19] G. Wheeler, J. McCoy and G. Williams, 'Lifespan theorem for constrained surface diffusion flows'. Math. Z. (2010), to appear.

\section{GLEN WHEELER, School of Mathematics and Applied Statistics, University of Wollongong, NSW 2522, Australia \\ e-mail: gw@gew75.com}

